\title{
Caracterización física y mecánica de los morteros antiguos. Aplicación a la evaluación del estado de conservación
}

\section{Physical and mechanical characterisation of historic mortars. Application to the evaluation of the state of conservation}

\author{
$\underline{\text { A. Magalhães }}^{(*)}$, R. Veiga
}

Recepción/Received: 17-V-07

Aceptación/Accepted: 16-VI-08

Publicado online/Online publishing: 3-VIII-09

\section{RESUMEN}

El conocimiento de las características físicas y mecánicas de los morteros antiguos es muy importante a la hora de seleccionar morteros de reparación y de planear adecuadamente cualquier intervención de reparación. Así, se hace necesario definir los métodos de ensayo de caracterización aplicables a las muestras irregulares y friables para evaluar dichas características.

En el presente trabajo se presentan y analizan algunos resultados de caracterización experimental de morteros antiguos portugueses, y se describen los métodos de ensayo utilizados para evaluar el comportamiento al agua y la resistencia a la compresión. Mediante la comparación con los resultados obtenidos en los ensayos con morteros nuevos, usando dichos métodos y también los métodos recomendados por la norma europea, se sacan conclusiones sobre el potencial de los métodos.

Por último, se establece una correlación entre los resultados experimentales y el nivel de deterioro inicial atribuido a los morteros antiguos mediante inspección visual.

Palabras-clave: mortero antiguo, ensayo, resistencia a compresión, capilaridad, estado de conservación.

\section{SUMMARY}

An understanding of the physical and mechanical characteristics of historic mortars is of vital importance in the selection of repair mortars for historic buildings. As a result, a need has arisen for test methods that can be used to assess those characteristics in irregular, friable samples.

The present paper discusses the results of the experimental characterization of historic Portuguese mortars. The test methods used to evaluate water resistance and compressive strength are described. The compressive strength values found for new mortars using the method proposed and the method specified in European standards are compared and the results used to draw conclusions on the potential of the new technique.

The correlation between the initial degree of deterioration determined by visual inspection and the experimental data is discussed.

Keywords: historic mortar, test, compressive strength, capillarity, state of conservation.

(*) Laboratorio Nacional de Ingeniería Civil (Lisboa, Portugal). 


\section{INTRODUCCIÓN}

Por lo general, la intervención en los muros de los edificios antiguos requiere el uso de morteros de sustitución o reparación que sean duraderos y compatibles con las estructuras originales, por lo que su comportamiento físico y químico debe ser parecido al de los morteros existentes. En este sentido, es importante comprender las características químicas, físicas y mecánicas de los morteros antiguos (1-4).

Estas características también son importantes para evaluar el grado de deterioro del mortero, que resulta fundamental para definir las estrategias de reparación.

En lo que respecta al análisis químico de los morteros originales, diversos estudios $(2,5-10)$ se han centrado principalmente en la caracterización e identificación de sus componentes mediante sofisticadas técnicas, lo que ofrece datos rigurosos sobre los componentes minerales y los compuestos químicos que causan el deterioro. Sin embargo, la caracterización química y mineralógica no aporta los conocimientos suficientes para tomar decisiones trascendentes. Es fundamental disponer de información adicional sobre las características físicas y mecánicas de los morteros para comprobar si garantizan el comportamiento adecuado del mortero antiguo, así como para diseñar nuevos materiales, que han de ser compatibles con los materiales ya existentes en cuanto a estética y características químicas, mecánicas y físicas (2).

En los morteros antiguos (completamente carbonatados), el coeficiente de capilaridad sirve para medir la compacidad del mortero de enfoscado y, por tanto, su resistencia, ya que en los morteros de cal una buena compacidad suele estar relacionada con una buena resistencia. Suponiendo que el mortero ya esté totalmente carbonatado, un índice bajo de huecos y los valores correspondientes del coeficiente de capilaridad y de resistencia, permitirán hacerse una idea del estado de conservación de las muestras de mortero de enfoscado.

El estudio de las características físicas y mecánicas de los morteros ya existentes permite conocer las que deben reunir, en la medida de lo posible, los morteros de sustitución y proporciona datos para formular nuevas mezclas para los morteros de reparación.

Sin embargo, existen dificultades para su evaluación porque no siempre es posible extraer una muestra lo suficientemente grande de mortero antiguo. Por otra parte, las muestras suelen ser inadecuadas para los ensayos físicos y mecánicos normalizados debido a su baja cohesión y a su forma irregular $(11,12)$.

En este trabajo se describen dos métodos de ensayo desarrollados para estos tipos de muestras, y se presentan

\section{INTRODUCTION}

As a rule, interventions on historic buildings call for durable replacement or repair mortars that are compatible with the original materials, i.e., with their physical and chemical properties. Consequently, an understanding of the chemical, physical and mechanical characteristics of historic mortars is imperative to intervention in such buildings (1-4).

These characteristics are also relevant to the assessment of mortar deterioration, which is essential to repair strategies.

A number of studies (2, 5-10) conducted with sophisticated laboratory techniques to characterize and indentify historic mortar components have provided reliable data on the mineral constituents and chemical compounds that cause deterioration. Chemical and mineralogical composition does not provide sufficient grounds for fundamental decision-making, however. Further information on the physical and mechanical characteristics of mortars is indispensable, both to ascertain whether they ensure satisfactory performance of historic mortar and to determine whether they are suitable for use to design new mortar, which must be both aesthetically and functionally (chemically, mechanically and physically) compatible with the existing materials (2).

In historic, completely carbonated mortars, the capillarity coefficient is a measure of the compactness and therefore the strength of the rendering mortar, for in lime mortars compactness is usually associated with high strength. Assuming the mortar to be completely carbonated, the resulting low volume of voids, together with the respective capillarity coefficient and strength values, provide insight into the state of conservation of rendering mortar samples.

The characteristics to which replacement mortars should be designed, as far as possible, and the formulation of new repair mixes should be based on an understanding of the physical and mechanical properties of the existing mortars.

The difficulty arises, however, when the amount of historic mortar that can be obtained for samples is insufficient for a reliable assessment. Moreover, due to their poor cohesion and irregular shapes, such samples are often ill suited to running standard physical and mechanical tests $(11,12)$.

The present article describes two test methods developed to surmount these difficulties, and the 
y comentan los resultados obtenidos con muestras de morteros antiguos tomadas in situ. En el caso del método para analizar la resistencia a compresión, se emplea la comparación con los resultados obtenidos en los ensayos con morteros nuevos, usando dichos métodos y también los propuestos por las normas EN 1015-11 o 1015-18, para sacar conclusiones sobre el potencial de unos y otros.

Por último, se analizan los datos experimentales, se establece una correlación entre éstos y el grado de deterioro atribuido inicialmente mediante inspección visual, y se valora la utilidad de la caracterización física y mecánica en la evaluación del estado de conservación de los morteros antiguos.

\section{CASOS PRÁCTICOS}

Los casos prácticos seleccionados corresponden a edificios de los siglos IV a XVIII con morteros antiguos de cal. En varios casos, los morteros antiguos muestran una resistencia y durabilidad muy elevadas a los factores de
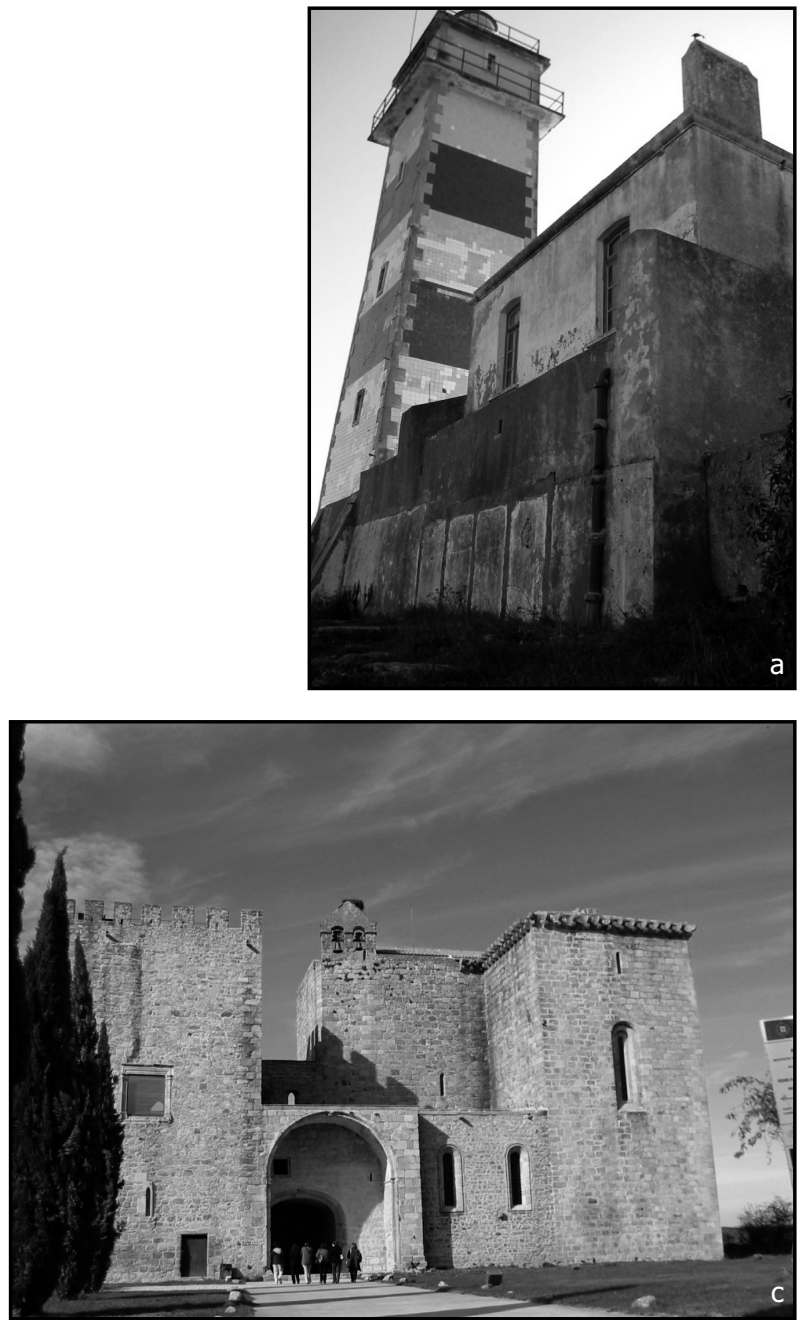

findings for samples taken from a number of historic buildings are discussed. The results of a comparative study of the compressive strength of new mortars found using the methods proposed and the method laid down in European standards EN 1015-11 and 1015-18 are used to draw conclusions on the potential of the new approach.

The experimental data are analyzed and correlated to the degree of deterioration assessed by visual inspection to determine the utility of physical and mechanical characterization in evaluating the state of conservation of historic mortars.

\section{CASE STUDIES}

The lime-based mortars chosen for the case studies were taken from buildings dating from the fourth to the eighteenth centuries. Several of these historic mortars proved to be highly weather-resistant and durable. The
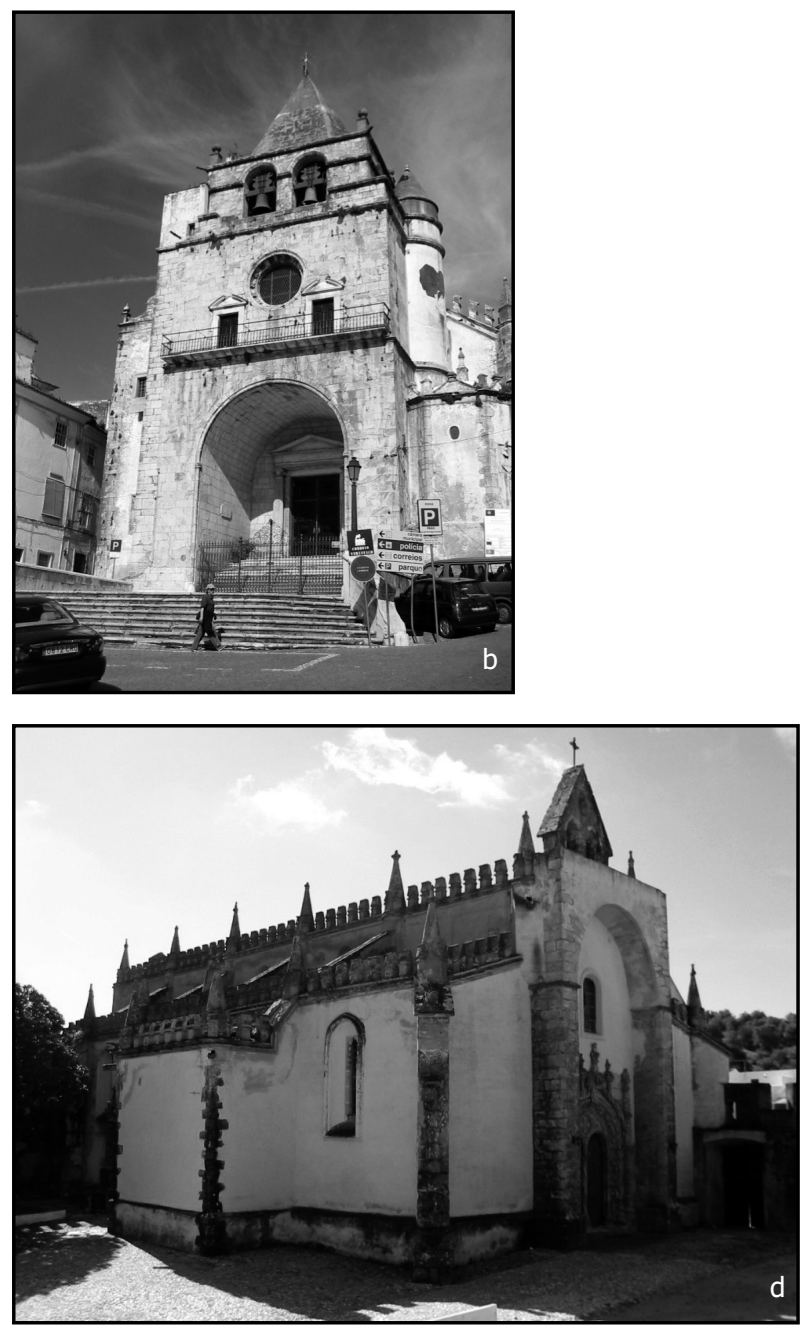

Figura 1. Algunos de los casos prácticos: a) faro de Santa Marta; b) iglesia de Elvas; c) santuario de Nossa Senhora da Boa Nova-Flor da Rosa; d) iglesia matriz de Mértola.

Figure 1. Sample case studies: a) Santa Marta Fortress; b) Elvas Church; c) Nossa Sra. da Boa Nova Sanctuary-Flor da Rosa; d) Mértola Church. 
meteorización, tanto físicos como químicos. Los defectos más graves de estos morteros son la pérdida de cohesión, las eflorescencias y criptoflorescencias, y la pérdida de adhesión.

Se atribuyó previamente a cada caso un grado de deterioro basándose en la inspección visual in situ del daño visible de los morteros, en función de los tipos de defectos observados y de su intensidad.

En la Tabla 1 se identifican los casos y morteros antiguos analizados, algunos de los cuales se recogen en la most serious flaws, found in some but not all of these mortars, included crumbling, efflorescence, cryptoflorescence and bonding loss.

The mortars were classified by level of deterioration based on in situ visual inspections, adjusted for type of flaw and intensity.

The case studies and historic mortars studied are listed in Table 1, and some are depicted in Figure 1. Photographs

Tabla 1 / Table 1

Características de los morteros antiguos.

Characteristics of historic mortars.

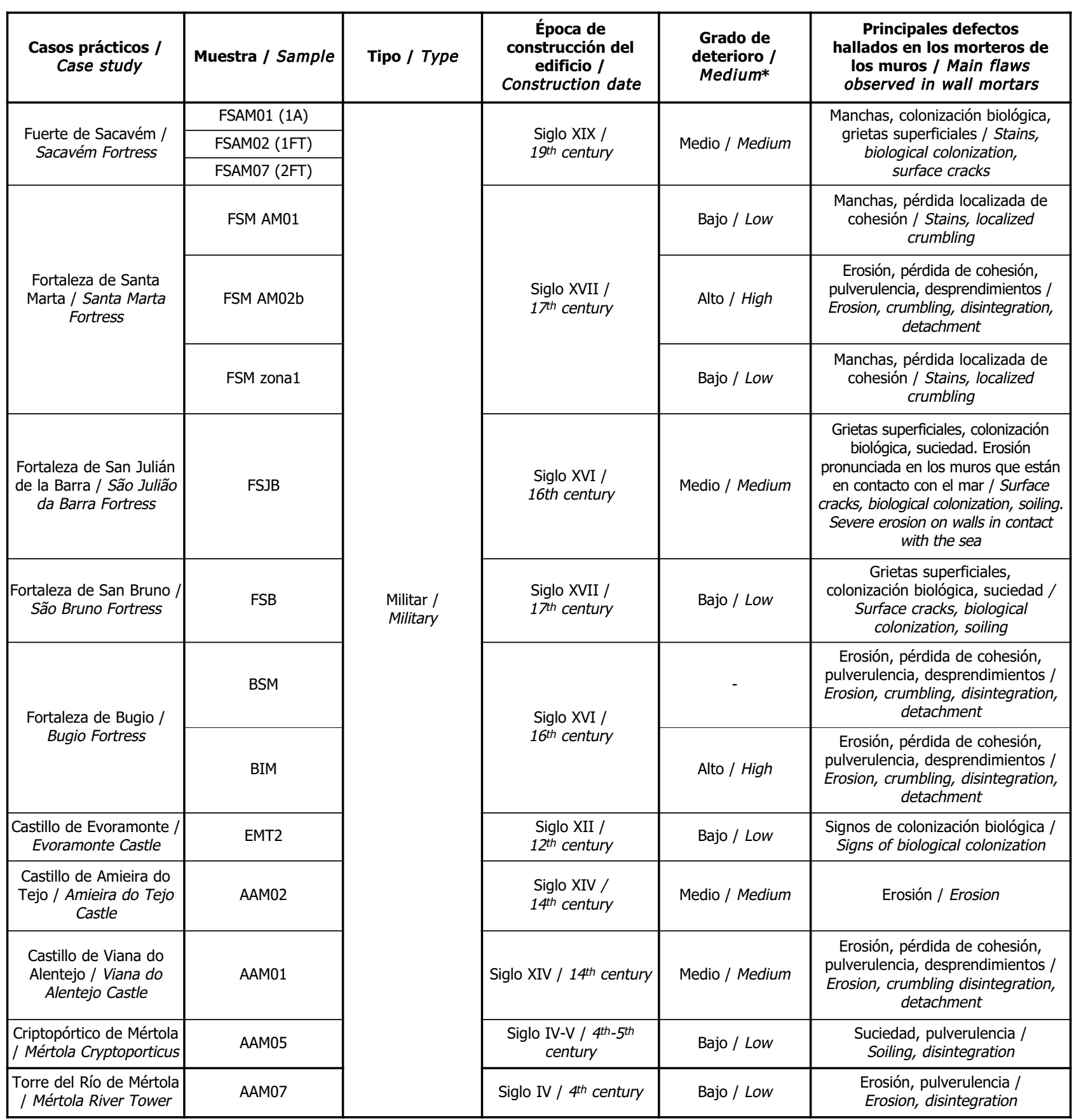


Figura 1. En la Figura 2 se presentan algunas de las muestras de mortero antiguo analizadas, con distintos grados de deterioro. of some of the mortar samples with different degrees of deterioration are reproduced in Figure 2.

Tabla 1 (cont.) / Table 1 (cont.)

Características de los morteros antiguos.

Characteristics of historic mortars.

\begin{tabular}{|c|c|c|c|c|c|}
\hline $\begin{array}{l}\text { Casos prácticos / } \\
\text { Case study }\end{array}$ & Muestra / Sample & Tipo / Type & $\begin{array}{c}\text { Época de } \\
\text { construcción del } \\
\text { edificio / } \\
\text { Construction date }\end{array}$ & $\begin{array}{l}\text { Grado de } \\
\text { deterioro / } \\
\text { Medium* }\end{array}$ & $\begin{array}{l}\text { Principales defectos } \\
\text { hallados en los morteros de } \\
\text { los muros / Main flaws } \\
\text { observed in wall mortars }\end{array}$ \\
\hline $\begin{array}{c}\text { Santa Casa de la } \\
\text { Inquisición de Monsaraz } \\
\text { / Santa Casa da } \\
\text { Inquisição at Monsaraz }\end{array}$ & SCI AM06 & \multirow{7}{*}{$\begin{array}{l}\text { Religioso / } \\
\text { Religious }\end{array}$} & $\begin{array}{l}\text { Siglo XVII / } \\
17^{\text {th }} \text { century }\end{array}$ & Bajo / Low & $\begin{array}{l}\text { Erosión, pulverulencia / } \\
\text { Erosion, disintegration }\end{array}$ \\
\hline $\begin{array}{l}\text { Iglesia de Elvas / } \\
\text { Elvas Church }\end{array}$ & AAM04 & & $\begin{array}{l}\text { Siglo XVI / } \\
16^{\text {th }} \text { century }\end{array}$ & - & - \\
\hline $\begin{array}{c}\text { Capilla de Amieira do } \\
\text { Tejo / Amieira do Tejo } \\
\text { Chapel }\end{array}$ & ATe - ATi & & $\begin{array}{l}\text { Siglo XVI / } \\
16^{\text {th }} \text { century }\end{array}$ & Alto / High & $\begin{array}{c}\text { Manchas de humedad, } \\
\text { subflorescencias, pérdida de } \\
\text { cohesión y adhesión, suciedad / } \\
\text { Damp stains, subflorescence, } \\
\text { crumbling, bonding loss, soiling }\end{array}$ \\
\hline $\begin{array}{l}\text { Iglesia matriz de Viana } \\
\text { do Alentejo / Viana do } \\
\text { Alentejo Church }\end{array}$ & MAT1A -MAT1B & & $\begin{array}{l}\text { Siglo XVI / } \\
16^{\text {th }} \text { century }\end{array}$ & - & - \\
\hline $\begin{array}{l}\text { Iglesia de la Misericordia } \\
\text { de Viana do Alentejo / } \\
\text { Misericórdia Church at } \\
\text { Viana do Alentejo }\end{array}$ & MIS3A-MIS1 & & $\begin{array}{l}\text { Siglo XVI / } \\
16^{\text {th }} \text { century }\end{array}$ & Alto / High & $\begin{array}{l}\text { Heterogeneidad del enlucido, } \\
\text { pérdida de cohesión y adhesión, } \\
\text { manchas de humedad, } \\
\text { eflorescencias / Plaster } \\
\text { heterogeneity, crumbling, bonding } \\
\text { loss, damp stains, efflorescence }\end{array}$ \\
\hline $\begin{array}{l}\text { Santuario de Nossa } \\
\text { Senhora da Boa Nova - } \\
\text { Flor da Rosa / Nossa } \\
\text { Sra. da Boa Nova } \\
\text { Sanctuary- } \\
\text { Flor da Rosa }\end{array}$ & AAM03 & & $\begin{array}{l}\text { Siglo XVII / } \\
17^{\text {th }} \text { century }\end{array}$ & Bajo / Low & $\begin{array}{l}\text { Suciedad, colonización biológica / } \\
\text { Soiling, biological colonization }\end{array}$ \\
\hline $\begin{array}{l}\text { Iglesia matriz de Mértola } \\
\text { / Mértola Church }\end{array}$ & AAM06 & & $\begin{array}{l}\text { Siglo XII / } \\
12^{\text {th }} \text { century }\end{array}$ & Medio / Medium & $\begin{array}{l}\text { Manchas de humedad / } \\
\text { Damp stains }\end{array}$ \\
\hline $\begin{array}{l}\text { Palacio de Maiorca / } \\
\text { Maiorca Palace }\end{array}$ & PMAM04 & $\begin{array}{l}\text { Palacio / } \\
\text { Palace }\end{array}$ & $\begin{array}{l}\text { Siglo XVIII / } \\
18^{\text {th }} \text { century }\end{array}$ & Bajo / Low & $\begin{array}{c}\text { Contaminación salina, colonización } \\
\text { biológica / Salt contamination, } \\
\text { biological colonization }\end{array}$ \\
\hline \multirow{2}{*}{$\begin{array}{l}\text { Muro «pombalino»1 / } \\
\text { "Pombalina" Wall }\end{array}$} & P7 & \multirow{2}{*}{$\begin{array}{l}\text { Edificio } \\
\text { residencial / } \\
\text { Residential }\end{array}$} & $\begin{array}{l}\text { Siglo XVIII-XIX / } \\
18^{\text {th }}-19^{\text {th }} \text { century }\end{array}$ & - & \\
\hline & P5 & & $\begin{array}{l}\text { Siglo XVIII-XIX / } \\
18^{\text {th }}-19^{\text {th }} \text { century }\end{array}$ & - & - \\
\hline $\begin{array}{l}\text { Molino de Queluz / } \\
\text { Queluz Windmill }\end{array}$ & MSO AM03 & \multirow{5}{*}{$\begin{array}{l}\text { Industrial / } \\
\text { Industrial }\end{array}$} & $\begin{array}{l}\text { Desconocido / } \\
\text { Unknown }\end{array}$ & Bajo / Low & $\begin{array}{l}\text { Manchas, grietas superficiales, sin } \\
\text { colonización química ni biológica / } \\
\text { Stains, surface cracks, no chemica } \\
\text { or biological colonization }\end{array}$ \\
\hline \multirow{3}{*}{$\begin{array}{c}\text { Cetaria de Troia / } \\
\text { Cetaria de Troia }\end{array}$} & CT1 & & & \multirow{3}{*}{ - } & \multirow{3}{*}{$\begin{array}{l}\text { Manchas superficiales / } \\
\text { Surface stains }\end{array}$} \\
\hline & CT2 & & $\begin{array}{l}\text { Stglos } 1 \text {-vitury } \\
1^{\text {st }} 6^{\text {th }} \text { century }\end{array}$ & & \\
\hline & BCT & & & & \\
\hline $\begin{array}{l}\text { Acueducto de Águas } \\
\text { Livres / "Águas Livres" } \\
\text { Aqueduct }\end{array}$ & AAL & & $\begin{array}{l}\text { Siglo XVIII / } \\
18^{\text {th }} \text { century }\end{array}$ & Bajo / Low & - \\
\hline
\end{tabular}

1 El término "pombalino" hace referencia a un método de construcción desarrollado y empleado en Lisboa tras el grave terremoto de 1755 . Se caracteriza por muros con entramados de madera unidos a estructuras, también de madera, en el suelo. Estas construcciones son bastante resistentes a los terremotos por su estructura dúctil y solidaria. / "Pombalino", a construction system developed in Lisbon after the severe earthquake that severely damaged the city in 1755 , is characterized by walls with timber cross-bracing connected to wooden floor members. Such buildings are fairly earthquake-resistant, thanks to their ductile, inter-connected structure.

* Clasificación basada en inspección visual / Classification based on visual analysis.

(-) Sin deterioro / (-) No deterioration. 

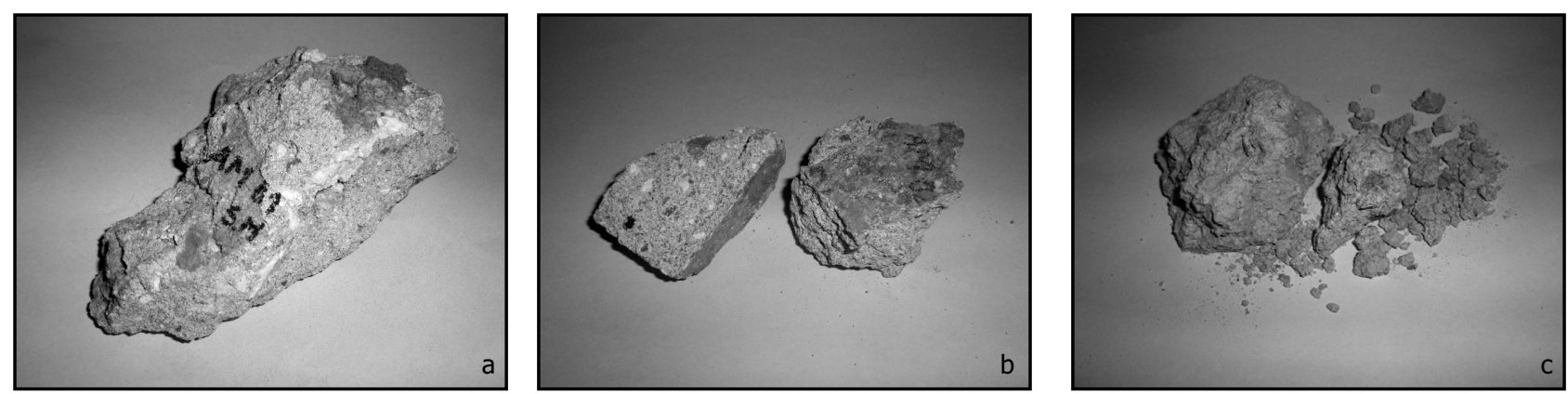

Figura 2. Muestras con distintos grados de deterioro: a) bajo; b) medio; c) alto.

Figure 2. Sample mortars showing different degrees of deterioration: a) low; b) medium; c) high.

\section{MÉTODOS DE ENSAYO}

Las muestras de enfoscado que pueden sacarse in situ suelen ser pequeñas e irregulares y, en ocasiones, tienen una baja cohesión, por lo que rara vez es posible aplicar directamente los ensayos normalizados existentes. Para superar este problema, se han estudiado y adaptado algunos métodos de ensayo especiales a las muestras de mortero antiguo recogidas en los edificios. Se prepararon dos ensayos -absorción capilar por contacto y resistencia a la compresión- y se hicieron experimentos con ellos en el LNEC junto a otros institutos europeos que participan en proyectos de investigación ${ }^{1}$. A continuación se ofrece un resumen de estos ensayos, que ya han sido calibrados y descritos en trabajos anteriores $(11,12)$ :

\subsection{Ensayo de absorción capilar por contacto (Figura 3)}

Consiste en pesar periódicamente las muestras, tras su colocación en una cesta, envueltas en una gasa geotextil2 húmeda, dentro de un recipiente transparente con agua. La muestra, que está en contacto con el agua a través del geotextil húmedo, se pesa junto con la cesta de alambre y la gasa húmeda cada 5 minutos durante los primeros 40 minutos y luego a intervalos de 60, 90, 180, 300, 480 y 1.440 minutos. El agua absorbida se determina por la diferencia entre el peso (cesta + gasa húmeda + muestra) medido en los distintos momentos y el peso inicial. El coeficiente de capilaridad obtenido por este método se denomina "coeficiente de capilaridad por contacto" (Ccc) y se determina a los 5 minutos y entre los minutos 10 y 90 (norma EN 1015-18) (13) si se prolonga la parte recta de la curva obtenida cuando se representa la absorción en función de la raíz cuadrada del tiempo.

1 Proyecto Historical Mortars, en el que participan el Laboratório Nacional de Engenharia Civil (Portugal), la Universidad de Liubliana (Eslovenia) y el Instituto de Mecánica Teórica y Aplicada (República Checa).

2 La superficie de las muestras es friable, por lo que el método normalizado mediante inmersión directa en agua no es viable. La gasa geotextil se emplea para evitar la pérdida de partículas finas solubles en agua.

\section{TEST METHODS}

The samples of rendering mortar that can be removed from historic buildings are usually small and irregular and sometimes exhibit poor cohesion. Consequently, they can seldom be subjected to standard testing. Test methods specially adapted to such mortar samples have been developed. Two, capillary absorption on contact and compressive strength, were prepared and calibrated at LNEC and other European institutes ${ }^{1}$, as reported in previous papers $(11,12)$ and summarized below.

\subsection{Capillary absorption on contact (Figure 3)}

This test consists in periodically weighing samples laid on a damp geotextile gauze ${ }^{2}$ inside a wire basket, in turn placed in a transparent pan containing water. This assembly, i.e., specimen, damp gauze and wire basket, is weighed every five minutes during the first 40, and then at intervals of 60,90,180,300, 480 and 1440 minutes. The amount of water absorbed is determined as the difference between the weight at time $t$ and the initial weight. The capillarity coefficient obtained, referred to here as the capillarity coefficient on contact $(\mathrm{CcC})$, is determined at five minutes and between $t=10$ and $t=90$ minutes (EN 1015-18) (13) when the straight part of the absorption versus square root of time curve extends into that interval.

1 Historical mortars Project, in which the Laboratório Nacional de Engenharia Civil (Portugal), University of Ljubljana (Slovenia) and Institute of Theoretical and Applied Mechanics (Czech Republic) participated.

2 Standard direct immersion is not feasible with these samples because their surfaces are friable. The geotextile gauze is used to prevent fine, water-soluble particles from coming loose. 


\subsection{Ensayo de resistencia a la compresión (Figura 4)}

Las muestras de mortero extraídas se colocan en un molde especial, dentro de un mortero resistente ("mortero de confinamiento"), que tiene la forma regular necesaria para poder usar la máquina de compresión que se emplea en el método especificado por la norma EN 1015-11 (14). La muestra puede sobresalir por los laterales del mortero de confinamiento, como puede verse en la Figura 4. Este "mortero de confinamiento", concebido para ser más resistente que las muestras, se aplica fresco sobre éstas y suele estar compuesto de cemento y arena silícea con una relación de 1:3 en peso (CEM II 32,5: arena). A continuación se realiza un ensayo de compresión directa, en el que se obtienen los valores de resistencia a compresión $\left(\mathrm{Sc}_{\mathrm{cm}}\right)$. Se emplea una prensa electromecánica ETIHM-S con una célula de carga de 200 kN.

Las muestras de mortero se someten primero al ensayo de absorción de agua y luego se secan y preparan para el ensayo de resistencia a compresión.

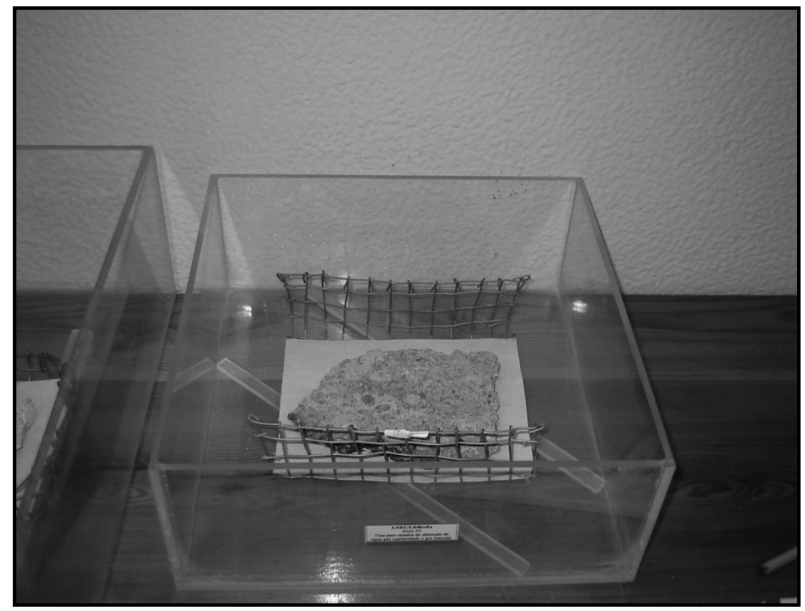

\subsection{Compressive strength (Figure 4)}

The mortar samples are placed in a mould to which a strong fresh mortar ("confinement mortar") is added to obtain the regular shape required to conduct the standard trial described in EN 1015-11 (14). The samples may protrude beyond the bounds of the confinement mortar, as shown in Figure 4. The fresh confinement mortar, designed to be stronger than the extracted samples, is normally made of CEM II 32.5 cement and siliceous sand at a ratio by weight of 1:3. The compression test is then conducted to find the respective strength values $\left(\mathrm{Sc}_{\mathrm{cm}}\right)$. In this case, an ETIHM-S testing machine with a 200-kN load cell was used for this purpose.

In the present study, the same samples were used for both tests, first water absorption, naturally, and once $d r y$, for compressive strength.

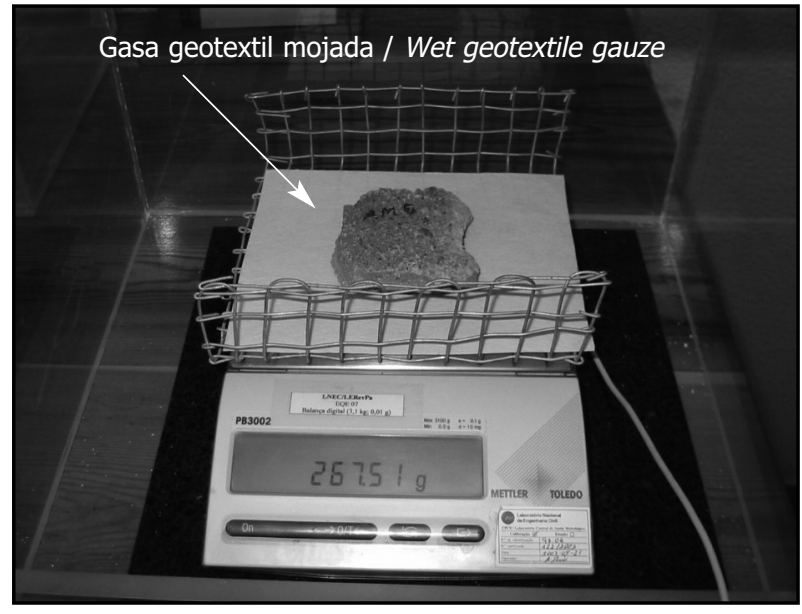

Figura 3. Ensayo de absorción capilar por contacto. Figure 3. Capillary absorption on contact.
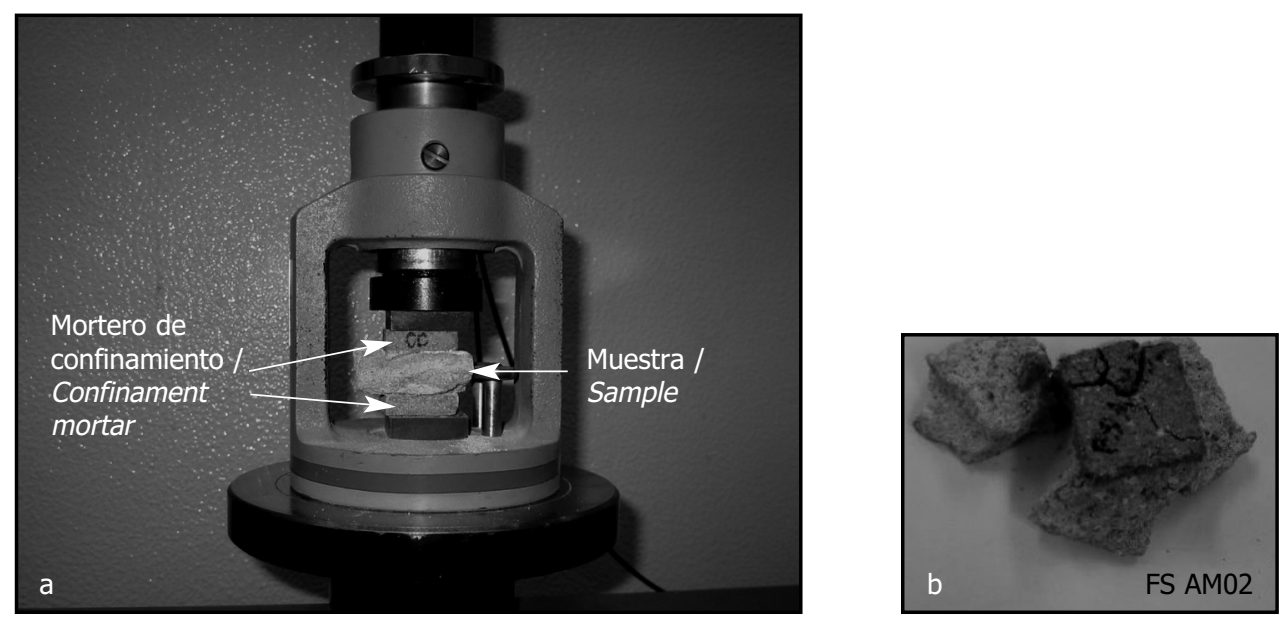

Figura 4. Morteros antiguos: a) ensayo de resistencia a compresión; b) muestra tras el ensayo de resistencia a compresión. Figure 4. Historic mortars: a) compressive strength test; b) sample after compressive strength test. 


\section{RESULTADOS DE LOS ENSAYOS}

Para evaluar la influencia de los "morteros de confinamiento" sobre los resultados obtenidos con los morteros antiguos, se realizaron ensayos con morteros de cemento y arena silícea con dos relaciones cemento: arena (en peso) distintas ( $1: 2$ y 1:3). Los resultados a los 28 días muestran que los valores de resistencia a compresión de estos morteros de cemento son mayores que los de los morteros antiguos $\left(21 \mathrm{~N} / \mathrm{mm}^{2}\right.$ para CEM $1: 2$ y $11 \mathrm{~N} / \mathrm{mm}^{2}$ para CEM $1: 3)$, lo que confirmó que los morteros de confinamiento son más resistentes que las muestras de mortero antiguo extraídas. Los resultados obtenidos con ambas composiciones confirman también que el mortero de confinamiento no influyó en los resultados de los morteros antiguos.

En la Tabla 2 se identifican los morteros de referencia preparados en el laboratorio y se presentan los resultados de los ensayos de resistencia a compresión realizados mediante los métodos tanto normalizados como adaptados. Para dar cabida a la representatividad de los nuevos morteros y obtener resultados más concluyentes, se analizaron distintas composiciones de morteros, consideradas como posibles soluciones para la reparación del enfoscado de muros (Figura 5).

\section{TEST RESULTS}

Cement-siliceous sand confinement mortars with different cement: sand ratios (1:2 and 1:3) were prepared and tested to determine their effect on the historic mortar strength findings. The 28-day compressive strength values of these mortars proved to be higher than the historic mortars (CEM 1:2-21N/mm² and CEM 1:3-11N/mm²). The results also confirmed that the confinement mortar had no impact on the strength values found for the historic mortars.

Comparison (new) mortars were prepared in the laboratory and tested for compressive strength using the standard and adapted methods. The results are given in Table 2. A number of mortars with different compositions, regarded to be possible repair mortars for historic buildings, were tested to this end (Figure 5).
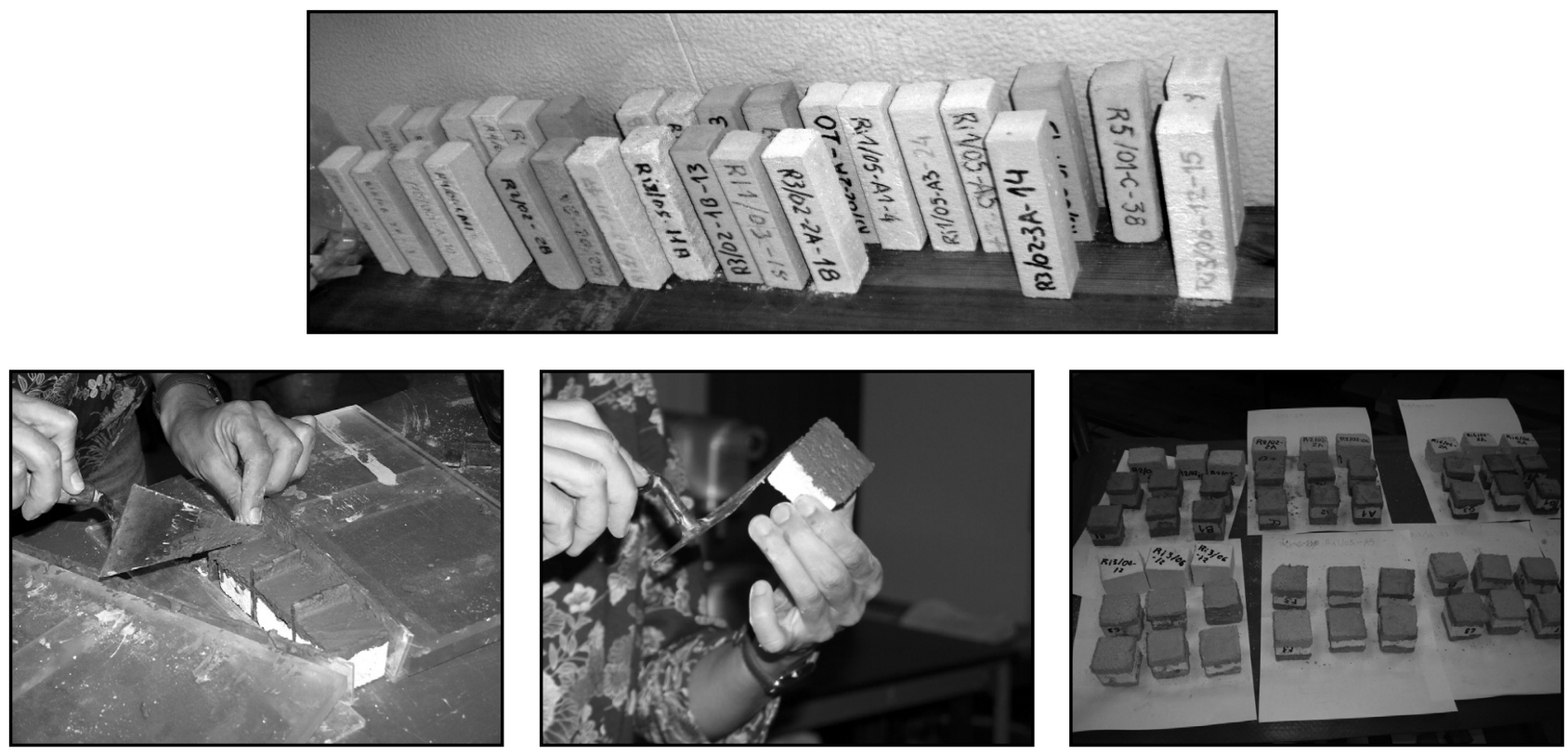

Figura 5. Probetas de mortero de cal preparadas para el ensayo de resistencia a compresión (método para muestras irregulares). Figure 5. Lime mortar specimens prepared for compressive strength testing (adaptation for irregular samples).

Los valores de resistencia a compresión obtenidos con los morteros de laboratorio mediante el método normalizado muestran desviaciones típicas bajas (DT < 0,11), mientras que se observó una mayor dispersión de los resultados obtenidos con las distintas probetas del mismo mortero con el método del confinamiento. Sin
The compressive strength values found for the laboratory mortars using the standard method had low standard deviations $(S D<0.11)$, while the dispersion was greater for specimens tested using the confinement method. Even in the latter, however, the $S D$ values were under 0.4 except in 
embargo, incluso en estos casos los valores de DT fueron inferiores a 0,4, a excepción de la probeta ALB950, cuya DT fue de 0,6 . specimen ALB950, where a value of 0.6 was observed.

Tabla 2 / Table 2

Valores de resistencia a la compresión de los morteros preparados en el laboratorio. Laboratory mortar compressive strength values.

\begin{tabular}{|c|c|c|c|c|c|c|c|c|c|c|c|}
\hline \multirow{4}{*}{$\begin{array}{l}\text { Mortero / } \\
\text { Mortar }\end{array}$} & \multirow{4}{*}{$\begin{array}{c}\text { Dosifica- } \\
\text { ción / } \\
\text { Volumetric } \\
\text { dosage }\end{array}$} & \multirow{4}{*}{$\begin{array}{l}\text { Componentes / } \\
\text { Constituents }\end{array}$} & \multicolumn{8}{|c|}{$\begin{array}{c}\text { Resistencia a la compresión }\left(\mathbf{N} / \mathbf{m m}^{2}\right) / \\
\text { Compressive Strength }\left(N / \mathrm{mm}^{2}\right)\end{array}$} & \multirow{4}{*}{$\begin{array}{c}\begin{array}{c}\text { Coeficiente de } \\
\text { capilaridad } \\
\left(\mathrm{kg} / \mathrm{m}^{2} \cdot \mathrm{h}^{1 / 2}\right) / \\
\text { Capillarity coeffi- } \\
\text { cient }\left(\mathrm{kg} / \mathrm{m}^{2} \cdot \mathrm{h}^{1 / 2}\right)\end{array} \\
\\
\text { Por contacto } \\
\text { Ccc (5min) / Contact } \\
\text { method } \\
\text { Ccc (5min) }\end{array}$} \\
\hline & & & \multicolumn{3}{|c|}{$\begin{array}{l}\text { Método normalizado / } \\
\text { Standard method }\end{array}$} & \multicolumn{4}{|c|}{$\begin{array}{c}\text { Método de mortero de } \\
\text { confinamiento / Confinement } \\
\text { mortar method }\end{array}$} & \multirow{3}{*}{$\begin{array}{l}\mathrm{Sc}_{\mathbf{s}} / \\
\mathrm{Sc}_{\mathrm{cm}}\end{array}$} & \\
\hline & & & \multirow{2}{*}{$\begin{array}{c}\text { Anti- } \\
\text { güedad } \\
\text { / Age }\end{array}$} & \multirow{2}{*}{$\mathrm{Sc}_{\mathbf{s}}$} & \multirow{2}{*}{$\begin{array}{l}\mathrm{DT} / \\
S D\end{array}$} & \multirow{2}{*}{$\begin{array}{l}\text { Anti- } \\
\text { güedad } \\
\text { / Age }\end{array}$} & \multicolumn{2}{|c|}{$\mathrm{Sc}_{\mathrm{cm}}$} & \multirow{2}{*}{$\begin{array}{c}\text { DT I } \\
S D\end{array}$} & & \\
\hline & & & & & & & $1: 2(2)$ & $1: 3$ & & & \\
\hline AL1 & \multirow{3}{*}{$1: 3$} & $\begin{array}{c}\text { Cal:arena de río con } \\
\text { granulometría idónea / Lime: } \\
\text { well graded river sand }\end{array}$ & $\begin{array}{c}90 \\
\text { días } / d\end{array}$ & 1.3 & 0.04 & $\begin{array}{c}3 \text { años/ } \\
\text { years }\end{array}$ & 1.5 & 2.0 & 0.4 & 0.7 & 12.7 \\
\hline AL2 & & $\begin{array}{l}\text { Cal:mezcla de arena de la zona } \\
\text { de Lisboa / Lime: mixed sand } \\
\text { from the Lisbon region }\end{array}$ & 1 año & 1.3 & 0.15 & $\begin{array}{l}1 \text { año/ } \\
\text { years }\end{array}$ & 1.2 & 1.3 & 0.2 & 1.1 & 12.2 \\
\hline AL3 & & $\begin{array}{c}\text { Cal:arena de río / Lime: river } \\
\text { sand }\end{array}$ & $\begin{array}{c}90 \\
\text { días/d }\end{array}$ & 0.8 & 0.05 & $\begin{array}{c}4 \text { años/ } \\
\text { years }\end{array}$ & 1.5 & 1.1 & 0.3 & 0.6 & 10.9 \\
\hline ALCe & $1: 3: 12$ & $\begin{array}{c}\text { Cemento:cal:arena de río de } \\
\text { granulometría idónea / } \\
\text { Cement: Lime: well graded } \\
\text { river sand }\end{array}$ & $\begin{array}{c}90 \\
\text { días/d }\end{array}$ & 1.9 & 0.07 & $\begin{array}{c}3 \text { años/ } \\
\text { years }\end{array}$ & ND & 2.5 & - & 0.8 & 15.2 \\
\hline ALPZ & $1: 0.5: 2.5$ & $\begin{array}{l}\text { Cal:puzolana:arena de río de } \\
\text { granulometría idónea / Lime: } \\
\text { pozzolan: graded river sand }\end{array}$ & $\begin{array}{c}90 \\
\text { días/d }\end{array}$ & 1.8 & 0.07 & $\begin{array}{c}3 \text { años } / \\
\text { years }\end{array}$ & 2.0 & 1.9 & 0.1 & 0.9 & 10.2 \\
\hline ALB950 & $1: 1: 4$ & $\begin{array}{l}\text { Cal:ladrillo }\left(950^{\circ}\right) \text { :arena } \\
\text { de río / Lime: brick }\left(950^{\circ}\right) \text { : } \\
\text { river sand }\end{array}$ & $\begin{array}{c}5 \\
\text { años/d }\end{array}$ & 0.8 & 0.07 & $\begin{array}{c}5 \text { años/ } \\
\text { years }\end{array}$ & 1.7 & 0.8 & 0.6 & 0.7 & 12.3 \\
\hline ALB750 & $1: 1: 4$ & $\begin{array}{l}\text { Cal:Iadrillo }\left(750^{\circ}\right) \text { :arena } \\
\text { de río / Lime: brick }\left(750^{\circ}\right) \text { : } \\
\text { river sand }\end{array}$ & $\begin{array}{c}90 \\
\text { días/d }\end{array}$ & 1.0 & 0.02 & $\begin{array}{c}5 \text { años } / \\
\text { years }\end{array}$ & 1.4 & 1.3 & 0.1 & 0.7 & 12.2 \\
\hline ALM & $1: 3$ & $\begin{array}{c}\text { Cal:arena de río: } 10 \% \\
\text { metacaolín (en peso de la cal) } \\
\text { / Lime: river sand: } 10 \% \\
\text { metakaolin (by lime weight) }\end{array}$ & $\begin{array}{c}2 \text { años/ } \\
\text { years }\end{array}$ & 0.4 & 0.04 & $\begin{array}{c}2 \text { años } / \\
\text { years }\end{array}$ & ND & 0.7 & - & 0.6 & 12.3 \\
\hline AHL1 & $1: 3$ & $\begin{array}{l}\text { Cal hidráulica artificial:mezcla } \\
\text { de arena de la zona de Lisboa } \\
\text { / Artificial hydraulic lime:mixed } \\
\text { sand from the Lisbon region }\end{array}$ & $\begin{array}{c}90 \\
\text { días/d }\end{array}$ & 3.1 & 0.11 & $\begin{array}{c}2 \text { años } / \\
\text { years }\end{array}$ & ND & 3.8 & - & 0.8 & 11.9 \\
\hline AHL 2 & $1: 1: 6$ & $\begin{array}{l}\text { Cal hidráulica artificial: cal } \\
\text { :mezcla de arena de la zona } \\
\text { de Lisboa / Artificial hydraulic } \\
\text { lime: Lime: mixed sand from } \\
\text { the Lisbon region }\end{array}$ & $\begin{array}{l}90 \\
\text { días/d }\end{array}$ & 0.6 & 0.02 & $\begin{array}{l}1 \text { año/ } \\
\text { years }\end{array}$ & ND & 0.8 & - & 0.8 & 20.2 \\
\hline HFL & $1: 3$ & $\begin{array}{l}\text { Cal hidrófuga: mezcla de arena } \\
\text { de la zona de Lisboa / Water- } \\
\text { repellent lime: mixed sand } \\
\text { from the Lisbon region }\end{array}$ & $\begin{array}{c}90 \\
\text { días/d }\end{array}$ & 0.6 & 0.04 & $\begin{array}{c}2 \text { años/ } \\
\text { years }\end{array}$ & ND & 1.0 & - & 0.6 & 1.4 \\
\hline HFLM1 & $1: 2.5$ & $\begin{array}{l}\text { Cal hidrófuga : arena de río: } 10 \\
\% \text { de metacaolín (del peso de } \\
\text { la cal) / Water-repellent lime: } \\
\text { river sand: } 10 \% \text { metakaolin } \\
\text { (by lime weight) }\end{array}$ & $\begin{array}{c}2 \text { años } / \\
\text { years }\end{array}$ & 0.7 & 0.00 & $\begin{array}{c}2 \text { años / } \\
\text { years }\end{array}$ & 0.7 & 0.7 & 0.0 & 1.0 & 0.3 \\
\hline HFLM2 & $1: 2.5$ & $\begin{array}{l}\text { Cal hidrófuga: arena de río: } 25 \\
\% \text { de metacaolín (del peso de } \\
\text { la cal) / Water-repellent lime: } \\
\text { river sand: } 25 \% \text { metakaolin } \\
\text { (by lime weight) }\end{array}$ & $\begin{array}{c}2 \text { años } / \\
\text { years }\end{array}$ & 1.0 & 0.06 & $\begin{array}{c}2 \text { años / } \\
\text { years }\end{array}$ & 0.9 & 0.9 & 0.0 & 1.1 & 1.9 \\
\hline
\end{tabular}


Tabla 2 (cont.) / Table 2 (cont.)

Valores de resistencia a la compresión de los morteros preparados en el laboratorio.

Laboratory mortar compressive strength values.

\begin{tabular}{|c|c|c|c|c|c|c|c|c|c|c|c|}
\hline \multirow{4}{*}{$\begin{array}{c}\text { Mortero I } \\
\text { Mortar }\end{array}$} & \multirow{4}{*}{$\begin{array}{c}\text { Dosifica- } \\
\text { ción / } \\
\text { Volumetrid } \\
\text { dosage }\end{array}$} & \multirow{4}{*}{$\begin{array}{c}\text { Componentes I } \\
\text { Constituents }\end{array}$} & \multicolumn{8}{|c|}{$\begin{array}{c}\text { Resistencia a la compresión }\left(\mathrm{N} / \mathrm{mm}^{2}\right) / \\
\text { Compressive Strength }\left(\mathrm{N} / \mathrm{mm}^{2}\right)\end{array}$} & \multirow{4}{*}{ 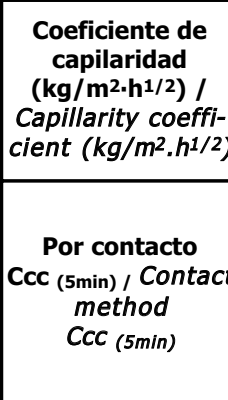 } \\
\hline & & & \multicolumn{3}{|c|}{$\begin{array}{l}\text { Método normalizado / } \\
\text { Standard method }\end{array}$} & \multicolumn{4}{|c|}{$\begin{array}{l}\text { Método de mortero de } \\
\text { confinamiento / Confinement } \\
\text { mortar method }\end{array}$} & \multirow{3}{*}{$\begin{array}{l}\mathrm{Sc}_{\mathbf{s}} / \\
\mathrm{Sc}_{\mathrm{cm}}\end{array}$} & \\
\hline & & & \multirow{2}{*}{\begin{tabular}{|c|} 
Anti- \\
güedad \\
$/$ Age
\end{tabular}} & \multirow{2}{*}{$\mathrm{Sc}_{s}$} & \multirow{2}{*}{$\begin{array}{l}\text { DT/ } \\
S D\end{array}$} & \multirow{2}{*}{$\begin{array}{c}\text { Anti- } \\
\text { güedad } \\
\text { / Age }\end{array}$} & \multicolumn{2}{|c|}{$\mathrm{Sc}_{\mathrm{cm}}$} & \multirow{2}{*}{$\begin{array}{l}\text { DT / } \\
S D\end{array}$} & & \\
\hline & & & & & & & $1: 2(2)$ & $1: 3$ & & & \\
\hline HFLM3 & $1: 2.5$ & $\begin{array}{l}\text { Cal hidrófuga: arena de río: } \\
25 \% \text { de metacaolín y } 5 \% \\
\text { de humo de sílice (del peso } \\
\text { de la cal) / Water-repellent } \\
\text { lime: river sand: } 20 \% \\
\text { metakaolin and } 5 \% \text { silica } \\
\text { fume (by lime weight) }\end{array}$ & $\begin{array}{c}2 \text { años } / \\
\text { years }\end{array}$ & 0.9 & 0.10 & $\begin{array}{c}2 \text { años } / \\
\text { years }\end{array}$ & 1.2 & 0.9 & 0.2 & 0.9 & 1.5 \\
\hline PD1 & - & $\begin{array}{l}\text { Mortero predosificado / Pre- } \\
\text { dosed mortar }\end{array}$ & $\begin{array}{c}90 \\
\text { días/d }\end{array}$ & 1.2 & 0.10 & $\begin{array}{c}2 \text { años } 1 \\
\text { years }\end{array}$ & 1.4 & 1.5 & 0.1 & 0.8 & 11.9 \\
\hline PD2 & - & $\begin{array}{c}\text { Mortero predosificado / Pre- } \\
\text { dosed mortar }\end{array}$ & $\begin{array}{c}90 \\
\text { días/d }\end{array}$ & 1.1 & 0.01 & $\begin{array}{c}3 \text { años } / \\
\text { years }\end{array}$ & 1.6 & 1.3 & 0.2 & 0.8 & 14.1 \\
\hline
\end{tabular}

(2) Valores comparativos; DT: desviación tipo; ND: no determinado / Comparison values; SD: standard deviation; Nd: not determined.

En general, las muestras recogidas tenían un grosor variable $y$, en algunos casos, estaban compuestas por más de una capa. En algunos casos no fue posible recoger más de una muestra debido a la débil cohesión, lo que limitó la representatividad de los resultados obtenidos en estos casos prácticos.

En la Tabla 3 se muestran los coeficientes de capilaridad por contacto y los resultados de resistencia mecánica obtenidos con las muestras de mortero antiguo.

\section{DISCUSIÓN DE LOS RESULTADOS}

\subsection{La resistencia a la compresión determinada mediante el método normalizado y el método del mortero de confinamiento}

La Figura 6 muestra la correlación entre la resistencia a compresión obtenida con el método normalizado $\left(\mathrm{Sc}_{\mathrm{s}}\right)$ y la obtenida con el método desarrollado para las muestras irregulares $\left(\mathrm{Sc}_{\mathrm{cm}}\right)$. La relación entre $\mathrm{Sc}_{\mathrm{s}}$ y $\mathrm{Sc}_{\mathrm{cm}}$ varía entre 0,6 y 1,0 , excepto en los casos AL2 y HFLM3, en los que la relación fue de 1,1 (Tabla 3). Esta variación puede considerarse aceptable si se tiene en cuenta que la resistencia del enfoscado previsiblemente aumentará con el tiempo. De hecho, es probable que cuando se realizaran los nuevos ensayos de resistencia a compresión a esas muestras, entre uno y tres años después de realizar el ensayo por el método normalizado, el grado de carbonatación de las muestras hubiera aumentado ya $\mathrm{y}$, por consiguiente, su resistencia fuera mayor y diera lugar a una relación $\mathrm{Sc}_{\mathrm{s}}-\mathrm{Sc}_{\mathrm{cm}}$ inferior a uno.
Sample thickness was uneven as a rule and in some cases comprised more than one layer. Moreover, at a number of sites only one sample could be collected due to the poor condition of the original mortar, reducing the representativeness of the findings in the respective case studies.

Table 3 gives the capillary coefficients on contact and compressive strength values for the historic mortars.

\section{DISCUSSION}

\subsection{Compressive strength determined by the standard and confinement mortar methods}

Figure 6 shows the correlation between the compressive strength obtained by the standard $\left(S c_{S}\right)$ and confinement $\left(S c_{c m}\right)$ methods. The $S c_{s} / S c_{c m}$ ratio, in turn, ranged from 0.6 to 1.0 except in two cases (AL2, HFLM3) where it was 1.1 (Table 3). This variation would appear to be acceptable, inasmuch as rendering mortar strength increases over time. Indeed, the confinement test was applied to the samples from one to three years after the standard test had been conducted. Since the degree of carbonation would logically have grown in the interim, $S c_{S} / S c_{C m}$ ratios would be expected to be lower than one. 
Tabla 3 / Table 3

Valores del coeficiente de capilaridad y de la resistencia a compresión de las muestras tomadas in situ. Historic mortar sample capillarity coefficient and compressive strength values.

\begin{tabular}{|c|c|c|c|c|}
\hline Casos prácticos / Case study & $\begin{array}{l}\text { Tipo / } \\
\text { Type }\end{array}$ & Muestra / Sample & $\begin{array}{c}\text { Coeficiente de capilaridad } \\
\text { Ccc5 }\left(\mathrm{kg} / \mathrm{m}^{2} \cdot \mathrm{h}^{1 / 2}\right) / \\
\text { Capillarity coefficient Ccc5 } \\
\left(\mathrm{kg} / \mathrm{m}^{2} \cdot \mathrm{h}^{1 / 2}\right)\end{array}$ & $\begin{array}{c}\text { Resistencia a la compresión } \\
\mathrm{Sc}_{\mathrm{cm}}\left(\mathrm{N} / \mathrm{mm}^{2}\right) / \\
\text { Compressive strength - } \\
S c_{c m}\left(\mathrm{~N} / \mathrm{mm}^{2}\right)\end{array}$ \\
\hline \multirow{3}{*}{ Fuerte de Sacavém / Sacavém Fortress } & \multirow{14}{*}{$\begin{array}{l}\text { Militar / } \\
\text { Military }\end{array}$} & FSAM01 & 5.4 & 1.2 \\
\hline & & FSAM02 & 27.9 & 1.8 \\
\hline & & FSAM07 & 14.7 & 3.0 \\
\hline \multirow{3}{*}{ Fortaleza de Santa Marta / Santa Marta Fortress } & & FSM AM01 & 5.7 & 2.4 \\
\hline & & FSM AM02b & 6.8 & 1.3 \\
\hline & & FSM zone1 & 3.4 & 3.6 \\
\hline $\begin{array}{l}\text { Fortaleza de San Julián de la Barra / São Julião da } \\
\text { Barra Fortress }\end{array}$ & & FSJB & 10.9 & 2.3 \\
\hline Fortaleza de San Bruno / São Bruno Fortress & & FSB & 1.4 & 7.1 \\
\hline \multirow{2}{*}{ Fortaleza de Bugio / Bugio Fortress } & & BSM & 9.1 & 1.8 \\
\hline & & BIM & 32.3 & 1.1 \\
\hline Castillo de Evoramonte / Evoramonte Castle & & EMT2 & $\mathrm{ND} / \mathrm{Nd}$ & 1.6 \\
\hline Castillo de Amieira do Tejo / Amieira do Tejo Castle & & AAM02 & 5.5 & 3.6 \\
\hline Criptopórtico de Mértola / Mértola Cryptoporticus & & AAM05 & 5.4 & 1.5 \\
\hline Torre del Río de Mértola / Mértola River Tower & & AAM07 & 2.3 & 3.5 \\
\hline $\begin{array}{l}\text { Santa Casa de la Inquisición de Monsaraz / Santa } \\
\text { Casa da Inquisição at Monsaraz }\end{array}$ & \multirow{11}{*}{$\begin{array}{c}\text { Religioso / } \\
\text { Religious }\end{array}$} & SCI AM06 & 13.2 & 2.5 \\
\hline Iglesia de Elvas / Elvas Church & & AAM04 & 5.0 & 5.9 \\
\hline \multirow{2}{*}{$\begin{array}{l}\text { Capilla de Amieira do Tejo / } \\
\text { Amieira do Tejo Chapel }\end{array}$} & & ATe & 7.6 & 1.2 \\
\hline & & ATi & 9.5 & 2.4 \\
\hline \multirow{2}{*}{$\begin{array}{l}\text { Iglesia matriz de Viana do Alentejo / Viana do } \\
\text { Alentejo Church }\end{array}$} & & MAT1B & 7.4 & 2.5 \\
\hline & & МАТЗВ & 6.3 & 1.6 \\
\hline \multirow{2}{*}{$\begin{array}{l}\text { Iglesia de la Misericordia de Viana do Alentejo / } \\
\text { Misericórdia Church at Viana do Alentejo }\end{array}$} & & MIS1 & 7.7 & 0.9 \\
\hline & & MIS3A & 20.0 & 0.8 \\
\hline $\begin{array}{l}\text { Castillo de Viana do Alentejo / Viana do Alentejo } \\
\text { Castle }\end{array}$ & & AAM01 & 10.5 & 2.4 \\
\hline $\begin{array}{c}\text { Santuario de Nossa Senhora da Boa Nova -Flor da } \\
\text { Rosa / Nossa Sra. da Boa Nova } \\
\text { Sanctuary - Flor da Rosa }\end{array}$ & & AAM03 & 2.3 & 1.9 \\
\hline Iglesia matriz de Mértola / Mértola Church & & AAM06 & 25.3 & 3.5 \\
\hline Palacio de Maiorca / Maiorca Palace & $\begin{array}{l}\text { Palacio / } \\
\text { Palace }\end{array}$ & PMAM04 & 8.1 & 2.1 \\
\hline \multirow{2}{*}{ Muro "pombalino" / "Pombalina" Wall } & \multirow{2}{*}{$\begin{array}{l}\text { Edificio } \\
\text { residencial / } \\
\text { Residential }\end{array}$} & P7 & 7.1 & 0.8 \\
\hline & & P5 & 10.2 & 1.0 \\
\hline Molino de Queluz / Queluz Windmill & \multirow{5}{*}{$\begin{array}{c}\text { Industrial / } \\
\text { Industrial }\end{array}$} & MSO AM03 & 9.4 & 2.2 \\
\hline \multirow{3}{*}{ Cetaria de Troia / Cetaria de Troia } & & CT1 & 17.2 & 2.5 \\
\hline & & СT2 & 3.3 & 4.5 \\
\hline & & BCT & 2.9 & 5.5 \\
\hline $\begin{array}{c}\text { Acueducto de Águas Livres / "Águas Livres" } \\
\text { Aqueduct }\end{array}$ & & AAL & 24.8 & 2.2 \\
\hline
\end{tabular}

ND: no determinado / Nd: not determined. 


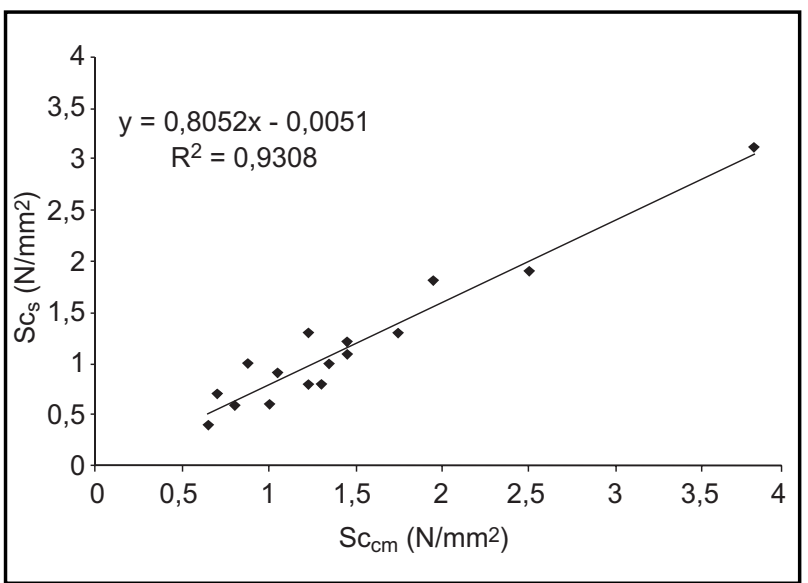

Figura 6. Morteros nuevos. Correlación entre la resistencia a compresión medida con el método normalizado y el nuevo método (muestras con mortero de confinamiento).

Figure 6. New mortars. Correlation between values obtained using the standard and confinement mortar methods.

\subsection{Ccc y propiedades mecánicas}

En las Figuras 7 y 8 se ha representado la relación entre el coeficiente de capilaridad a los 5 minutos (Ccc $5 \mathrm{~min}$ ) y la resistencia a compresión $\left(\mathrm{SC}_{\mathrm{cm}}\right)$ obtenidas, respectivamente, para los morteros nuevos y los antiguos.

En la Figura 7 no se incluyen los valores del mortero de cal hidrófugo, ya que la menor capilaridad de estos morteros no está relacionada con su compacidad y, por ello, no es posible relacionarla con su resistencia mecánica.

Los gráficos muestran que los morteros preparados en el laboratorio, que probablemente no estaban totalmente carbonatados, presentan coeficientes de capilaridad entre 10 y $20 \mathrm{~kg} / \mathrm{m}^{2} \cdot \mathrm{h}^{1 / 2}$ y valores de resistencia a la compresión que, en su mayoría, son inferiores a los $3 \mathrm{~N} / \mathrm{mm}^{2}$.

Una parte de los morteros antiguos estudiados, cada uno con un grado de deterioro distinto, muestra una distribución

\subsection{Ccc and mechanical characteristics}

The relationship between the 5-minute capillarity coefficient $\left(\mathrm{CCC}_{5}\right)$ and compressive strength $\left(\mathrm{Sc}_{\mathrm{cm}}\right)$ for new and historic mortars is shown in Figures 7 and 8, respectively.

The values for water-repellent lime mortar were excluded from the data in Figure 7, for their low capillarity is unrelated to their compactness and hence to mechanical strength.

According to these graphs, the laboratory mortars, which were probably not fully carbonated, had capillarity coefficients of from 10 to $20 \mathrm{~kg} / \mathrm{m}^{2} \cdot \mathrm{h}^{1 / 2}$ and compressive strengths of under $3 \mathrm{~N} / \mathrm{mm}^{2}$.

Some of the historic mortars with different degrees of deterioration exhibited a wider range of absorption rates

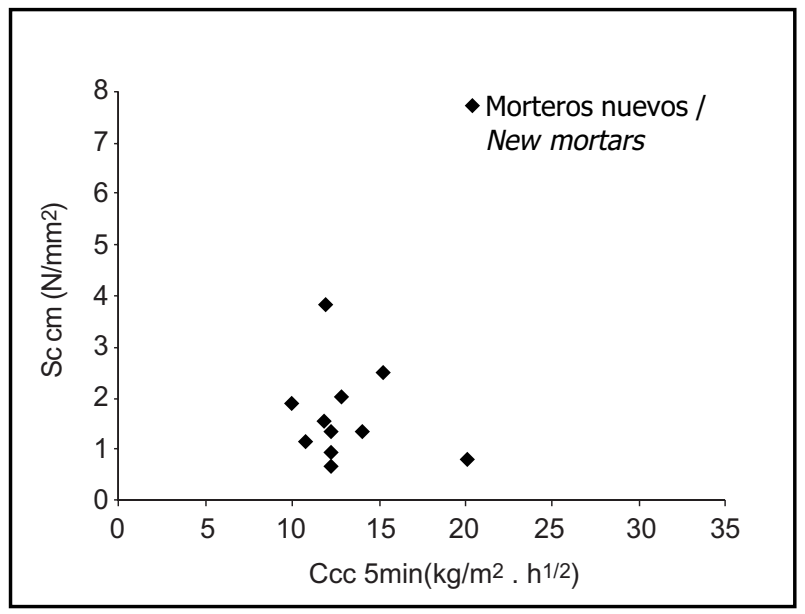

Figura 7. Morteros nuevos. Relación entre resistencia a compresión y coeficiente de capilaridad por contacto. Figure 7. New mortars. Compressive strength versus capillarity coefficient on contact. 
más amplia de los valores de velocidad de absorción y de resistencia a compresión. Estos resultados eran de esperar en estos morteros heterogéneos, con distintas características de antigüedad, nivel de deterioro, condiciones climáticas, requisitos de resistencia, etc.

Debería realizarse un análisis exhaustivo para relacionar el grado de deterioro con el coeficiente de capilaridad y los valores de resistencia a compresión. La información obtenida sería de utilidad para establecer recomendaciones de intervención. and compressive strength values. Such results could be expected from such heterogeneous mortars, whose age, level of deterioration, local climates, strength requirements and so on varied.

An in-depth analysis should be conducted to correlate deterioration to the capillarity coefficient and compressive strength values. The information obtained may prove to be useful for establishing recommendations for intervention.

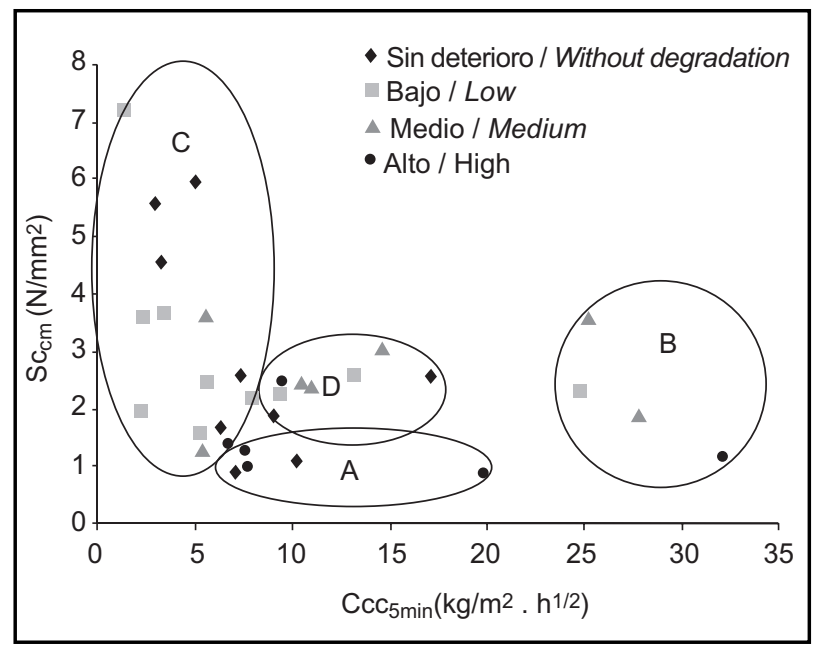

Figura 8. Morteros antiguos. Relación entre resistencia a la compresión y coeficiente de capilaridad por contacto. Figure 8- Historic mortars: Compressive strength versus capillarity coefficient on contact.

\subsection{Grado de deterioro, Ccc y propiedades mecánicas}

Las Figuras 9 y 10 muestran de forma esquemática la relación entre el grado inicial de deterioro de los distintos casos (Tabla 1) y los valores mínimo y máximo de los parámetros analizados: la resistencia a compresión y el coeficiente de capilaridad por contacto a los 5 minutos (Tabla 3). También se indican los valores medios (VM), la desviación típica (DT) y el coeficiente de variación (CV).

Los distintos grados de deterioro de los morteros y la gran variación entre los coeficientes de absorción capilar y los valores de resistencia a compresión obtenidos demuestran la escasa homogeneidad existente entre las muestras analizadas. En parte, esta conclusión era predecible si se tiene en cuenta la variedad de características de las muestras, tanto por el origen de los morteros como por su composición.

Los morteros con un grado de deterioro medio o alto muestran valores de resistencia a compresión más homogéneos. Respecto al coeficiente de capilaridad, la

\subsection{Level of deterioration, Ccc and mechanical characteristics}

Figures 9 and 10 show the range of 5-minute capillarity coefficient and compressive strength values (Table 3) for the degrees of deterioration defined (Table 1), along with the respective means (AV), standard deviations (SD) and coefficients of variation (CoV) values.

The variation in the degree of deterioration and the high CoV for both parameters are an indication of the low homogeneity in the samples analyzed. This conclusion was foreseeable, in light of the diversity of the origin and composition of the mortars studied.

Mortars with a medium or high degree of deterioration exhibited more uniform compressive strength values. The correlation between degree of deterioration and 
correlación entre el grado de deterioro y esta característica es difícil de determinar, debido a la gran variabilidad (> 57\%) de los resultados para todos los niveles de deterioro.

El análisis de las Figuras 9 y 10 permite observar además que los morteros con un grado elevado de deterioro muestran un Ccc5 más elevado y menor resistencia a compresión, mientras que los morteros con un grado de deterioro bajo muestran un Ccc5 más bajo y mayor resistencia a compresión. capillarity coefficient was difficult to establish, given the high (> 57\%) variability of the results for all levels of deterioration.

An analysis of Figures 9 and 10 also reveals the following: the most highly deteriorated mortars had the highest $\mathrm{CCC}_{5}$ and lowest compressive strength, while the least deteriorated materials had the lowest $\mathrm{CCC}_{5}$ and highest compressive strength.

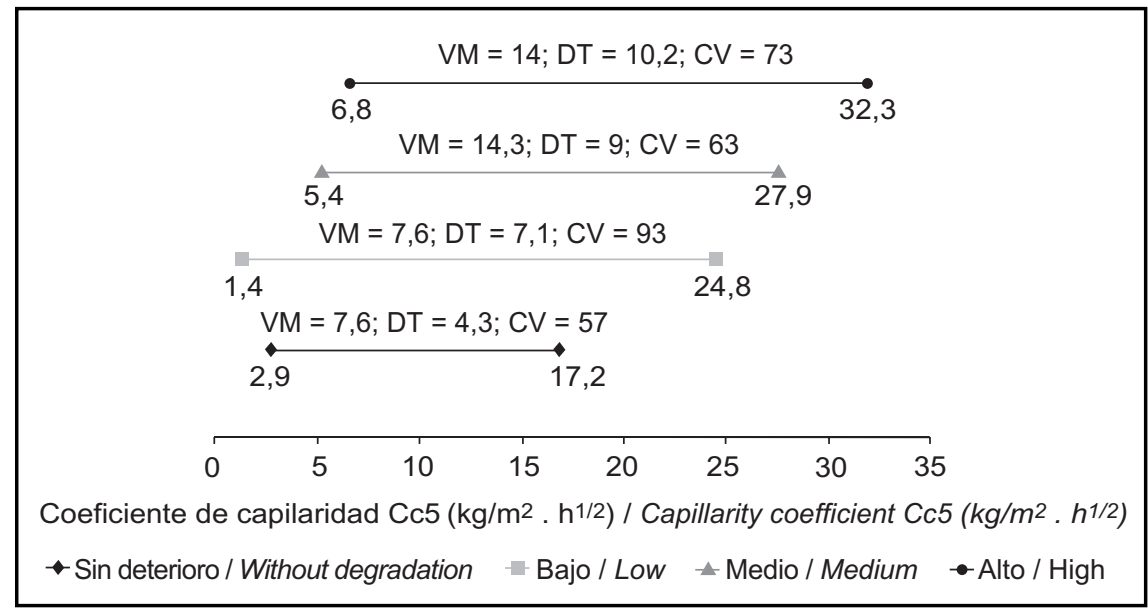

Figura 9. Representación esquemática de la relación entre grado de deterioro y coeficiente de capilaridad por contacto (Ccc5). Figure 9. Range of capillarity coefficients on contact $\left(\mathrm{CCC}_{5}\right)$ by level of mortar deterioration.

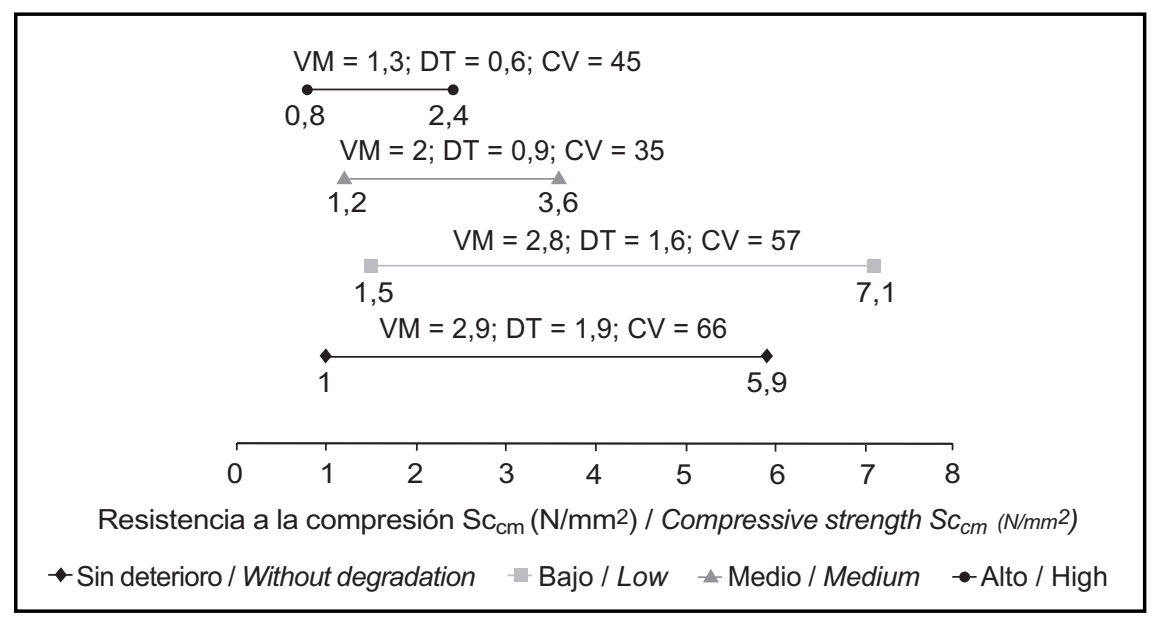

Figura 10. Representación esquemática de la relación entre grado de deterioro y resistencia a la compresión ( $\left.\mathrm{Sc}_{\mathrm{cm}}\right)$. Figure 10. Range of compressive strength $\left(\mathrm{SC}_{\mathrm{cm}}\right)$ by level of mortar deterioration.

\subsection{Análisis general}

Como ya se ha dicho, un análisis exhaustivo de los casos prácticos y la información obtenida resultan útiles para poder hacer recomendaciones sobre las posibles intervenciones.

\subsection{Global analysis}

As noted above, a detailed analysis of the case studies and the information obtained may be used to formulate recommendations for intervention. 
En la Figura 8 se definieron cuatro regiones $-\mathrm{A}, \mathrm{B}, \mathrm{C}$ y D- a partir de los grupos de resultados de los ensayos físicos y mecánicos.

Los valores más bajos de resistencia a compresión se obtuvieron con el muro "pombalino" y la iglesia de la Misericordia de Viana do Alentejo. En ambos casos se trata de morteros de yeso para interiores, que precisan de una menor resistencia que los morteros para enfoscados exteriores o para juntas. Sin embargo, en el segundo caso se trata de una muestra clasificada mediante inspección visual como muy deteriorada. La Figura 8 muestra estos casos, que se encuentran en la zona A. Los morteros exteriores de Amieira do Tejo también aparecen en esta zona, ya que tienen poca resistencia y fueron clasificados como muy deteriorados.

Las muestras de enfoscado FSAM02, BIM, AAL y AAM06 tienen coeficientes de capilaridad bastante elevados. Deberá estudiarse esta característica con mayor detenimiento para evaluar la necesidad de añadir enlucidos de protección a estos enfoscados. No obstante, puesto que estos morteros presentan una resistencia bastante buena, este tipo de medida deberá adoptarse únicamente si se detectan en el edificio problemas claramente relacionados con la falta de protección contra la humedad. Estos morteros se muestran en la zona B de la Figura 8.

En la zona C de la Figura 8, la mayoría de los casos corresponden a morteros que no presentan deterioro 0 con un grado de deterioro bajo, evaluación que justificaría el mantenimiento de estos materiales originales.

En la zona $\mathrm{D}$ se recogen morteros con características intermedias, parecidas a las prestaciones de los morteros de sustitución. En estos casos, el mantenimiento también sería una decisión lógica.

\section{CONCLUSIONES}

Es necesario tener información sobre las características físicas, mecánicas y químicas para poder adaptar la formulación de los morteros de reparación y para evaluar su estado de conservación.

El estudio de numerosos casos ha permitido evaluar la idoneidad de dos métodos de ensayo adaptados a muestras irregulares y friables con el fin de utilizarlos como técnicas auxiliares de evaluación del estado de conservación de los enfoscados antiguos.

De los resultados obtenidos puede concluirse que:
The points in the graph in Figure 8 were grouped into four regions, $A, B, C$ and $D$, defined on the grounds of physical and mechanical results.

The lowest compressive strength values were observed for the "pombalina" wall and Misericórdia Church at Viana do Alentejo. Both samples were taken from interior wall plastering, whose strength requirements are lower than for outdoor rendering or joint mortars. The latter sample, however, was visually classified to be highly deteriorated. Both are located in region $A$ in Figure 8. The Amieira do Tejo mortars were also included in this region in light of their low strength values and high degree of deterioration.

Rather high capillarity coefficients were observed for samples FSAM02, BIM, AAL and AAM06. This finding should be explored in greater depth to evaluate the advisability of applying a protective coating to the existing mortar. Since these mortars exhibited acceptable strength values, however, such measures should only be adopted if damp-related problems are clearly identified in the building. These mortars appear in region $B$ on Figure 8.

Since most of the mortars in region $C$ showed little or no deterioration, the decision to maintain the existing materials would be fully justified.

The performance recorded for all the remaining mortars, i.e., the samples in region $D$, was similar to the findings for the replacement mortars. Maintaining the original rendering would therefore be the logical decision for these mortars as well.

\section{CONCLUSIONS}

Information about the physical and mechanical, as well as the chemical properties of mortars is requisite to designing repair mortars and evaluating their state of conservation.

Two adapted test methods were analyzed on a large number of sample mortars to determine their suitability as supplementary techniques in the evaluation of the state of conservation of historic mortars.

The conclusions to be drawn from the findings are as follows: 
1. Aunque está claro que la determinación de la resistencia a compresión de las muestras irregulares de mortero con el método mencionado no proporciona un valor absoluto exacto, sí permite obtener valores con fines comparativos.

2. La composición de los "morteros de confinamiento" no influye en los resultados. No obstante, es precisa una calibración adicional para evaluar la influencia que el grosor de la muestra puede tener en los resultados.

3. Junto con el coeficiente de capilaridad por contacto a los 5 minutos, la resistencia a compresión puede ayudar a determinar el estado de conservación de los morteros antiguos como complemento a los resultados de la inspección visual.

4. Conviene destacar la importancia de realizar ensayos de caracterización física y mecánica para evaluar de forma objetiva el estado de conservación, ya que en algunos casos la inspección visual da lugar a clasificaciones erróneas.

5. La mayoría de las muestras analizadas en este trabajo presentaban valores de resistencia a compresión y coeficiente de capilaridad aceptables para su uso con los morteros formulados en el laboratorio para su aplicación en intervenciones en edificios antiguos. Los valores obtenidos y la inspección visual hacen recomendable la conservación de los morteros estudiados, posiblemente con alguna obra de reparación puntual, exceptuando tal vez los morteros de la iglesia de la Misericordia. En este caso, debe considerarse la posibilidad de tratamientos de consolidación o de sustitución parcial con morteros nuevos compatibles.

6. La comparación entre los resultados obtenidos con morteros de sustitución y las distintas zonas (A, B, C y D) obtenidas con los morteros antiguos corrobora la decisión de conservar los morteros antiguos situados en las zonas C y D. De lo contrario, podrían sustituirse los morteros antiguos por otros que no fueran compatibles o que tuvieran prestaciones parecidas o incluso inferiores.

7. Un mayor desarrollo de este estudio probablemente permita analizar de forma exhaustiva la influencia de la estructura porosa y la distribución del tamaño de poro en la resistencia mecánica y al agua de los morteros antiguos, así como establecer una relación entre las características de la estructura porosa y las características físicas y mecánicas.

\section{AGRADECIMIENTOS}

Este estudio se ha realizado en el marco de los proyectos de investigación "Desarrollo de metodologías para la evaluación de los efectos de la humedad en muros antiguos" (POCTI/ECM/46323/2002) y "CATHEDRAL - Caracterización y conservación de morteros históricos tradicionales de los edificios religiosos del Alentejo" (POCI/HEC/57915/2004), ambos financiados
1. Although the method proposed does not provided absolutely accurate compressive strength values for irregularly shaped mortar samples, it suffices for the purposes of comparison.

2. The confinement mortars used in this adapted test do not affect the results. Nonetheless, further calibration is necessary to assess their impact on the results in terms of sample thickness.

3. The 5-minute capillarity coefficient, together with compressive strength, can supplement visual analysis in the characterization of the state of conservation of historic mortars.

4. In any event, physical and mechanical tests are necessary for objective assessments of the state of conservation, for visual analysis alone may not suffice for accurate evaluation.

5. Most of the samples analyzed exhibited acceptable compressive strength and capillarity coefficient values compared to the laboratory mortars formulated for interventions in historic buildings. The values obtained, together with the visual inspection, evinced the need for conservation of the mortars studied and possible repair works in some cases. The Misercórdia Church mortars may constitute an exception, for it may require consolidation or partial replacement of the existing mortar with compatible new materials.

6. The comparison of the findings for the replacement mortars and the historic mortars in regions $A, B, C$ and $D$ corroborates the decision to preserve the historic mortars in the latter two categories. Indeed, the contrary may lead to the application of replacement mortars that are neither compatible nor more effective than the original material.

7. Further studies in this same line should address the effect of the pore structure and pore size distribution on the strength and water resistance of historic mortars and the correlation between the pore structure and physical and mechanical characteristics of these mortars.

\section{ACKNOWLEDGEMENTS}

This study was conducted under the projects titled "Development of methodologies for the assessment of moisture effects on historic structures" (POCTI/ECM/46323/2002) and "CATHEDRAL Characterisation and conservation of traditional historic mortars from religious buildings in Alentejo" (POCI/HEC/57915/2004), both funded by the 
por la Fundação para a Ciência e Tecnologia (Fundación para la Ciencia y la Tecnología) de Portugal. Los autores desean agradecer la contribución de Dora Santos, técnica del Departamento de Edificios del LNEC, por su valiosa ayuda en la preparación de las muestras y realización de los ensayos.
Portuguese "Fundação para a Ciência e Tecnologia" (Foundation for Science and Technology). The authors wish to thank Dora Santos of the LNEC Buildings Department for her invaluable assistance in specimen preparation and testing.

\section{BIBLIOGRAFÍA / BIBLIOGRAPHY}

(1) Moropoulou, A.; Polikreti, A.; Bakolas, A.; Michailidis, P.: "Correlation of physicochemical and mechanical properties of historical mortars and classification by multivariate statistics", Cem. Concr. Res., 33 (2003), pp. 891-898. doi:10.1016/S0008-8846(02)01088-8

(2) Veiga, M. R. et al.: "Methodologies for characterisation and repair of mortars of ancient buildings", in Proc. International Seminar of Historical Constructions 2001, Guimarães, Portugal, Universidade do Minho, 2001, pp.353-362.

(3) Lanas J., Álvarez: "Masonryrepair lime-based mortars: Factors affecting the mechanical behaviour", Cem. Concr. Res., 33 (2003), pp. 1867-1876. doi:10.1016/S0008-8846(03)00210-2

(4) Palomo, A.; Blanco-Varela, M. T.; Martínez-Ramírez, S.: "Historic Mortars: Characterization and Durability", New Tendencies for Research, p. 20 (http://www.arcchip.cz/w09/w09_palomo.pdf)

(5) Moropoulou, A.; Bakolas, A.; Anagnostopoulou, S.: "Composite materials in ancient structures", Cement and Concrete Composites 2005, 27, pp. 295-300. doi:10.1016/j.cemconcomp.2004.02.018

(6) Moropoulou, A; Bakolas, A; Bisbikou, K.: "Investigation of the technology of historic mortars", Journal of Cultural Heritage 2000 ; 1

(1), pp. 45-58. doi:10.1016/S1296-2074(99)00118-1

(7) Groot, C.; Bartos, P.; Hughes, J.: "Characterisation of old mortars with a respect to their repair", in Proc. 12th International Brick/Block Masonry Conference, Madrid (2000), p. 815-827.

(8) Santos Silva, A.; Ricardo, J.; Salta, M.; Adriano, P.; Mirão, J.; Candeias, A. E.: "Characterisation of roman mortars from the historical town of Mertola", in proc. Int. Conf. Heritage Weathering and Conservation, Madrid (2006), pp.85-90.

(9) Santos Silva, A.; Paiva, M.; Ricardo, J.; Salta, M.; Monteiro, A. M.; Candeias, A. E.: "Characterisation of roman mortars from the archaeological site of Troia", Mater. Sci. For., in press.

(10) Candeias, A. E.; Nogueira, P; Mirão, J.; Santos Silva, A., Veiga, R.; Casal, M. G.; Ribeiro, I.; Seruya, A. I.: "Characterization of ancient mortars: present methodology and future perspectives", in Proc. Workshop on Chemistry in the Conservation of Cultural Heritage: present and future perspectives, Perugia, (2006), 4 pp. (http://www.eu-artech.org/files/Ext_ab/candeias.pdf)

(11) Válek, J.; Veiga, R.: "Characterisation of mechanical properties of historic mortars - testing of irregular samples", in: Proc. 9th International Conference on Structural Studies, Repairs and Maintenance of Heritage Architecture (STREMAH, 2005), University of Malta, Malta (2005), $10 \mathrm{pp}$.

(12) Veiga, M. R.; Magalhães, A. C.; Bosilikov, V. B.: "Capillary tests on historic mortar samples extracted from site. Methodology and compared results", in Proc. 13th Int. Brick and Block Masonry Conference, Amsterdam (2004), 10 pp.

(13) European Committee of Standardization (CEN): "Methods of test for mortar for masonry - Part 18: Determination of water absorption coefficient due to capillary action of hardened rendering mortar", Brussels, April 1999, EN 1015-18.

(14) European Committee of Standardization (CEN): "Methods of test for mortar for masonry - Part 11: Determination of flexural and compressive strength of hardened rendering mortar", Brussels, August 1999, EN 1015-11. 